\title{
POLYNOMIAL APPROXIMATIONS OF FUNCTIONS WITH ENDPOINT SINGULARITIES AND PRODUCT INTEGRATION FORMULAS
}

\author{
GIUSEPPE MASTROIANNI AND GIOVANNI MONEGATO
}

\begin{abstract}
Several problems of mathematical physics lead to Fredholm integral equations of the second kind where the kernels are either weakly or strongly singular and the known terms are smooth. These equations have solutions which are smooth in the whole interval of integration except at the endpoints where they have mild singularities. In this paper we derive new pointwise and uniform polynomial approximation error estimates for that type of function. These estimates are then used to obtain bounds for the remainder terms of interpolatory product rules, based on the zeros of classical Jacobi orthogonal polynomials, that have been proposed for the discretization of integrals of the form
\end{abstract}

$$
\int_{-1}^{1} k(x, y) f(x) d x
$$

appearing in the integral equations mentioned above.

\section{INTRODUCTION}

Several problems of mathematical physics lead to Fredholm integral equations of the second kind,

$$
u(y)+\lambda \int_{-1}^{1} k(x, y) u(x) d x=h(y),
$$

where the kernel $k(x, y)$ is either weakly or strongly singular. For a few examples of such equations see $[1,6,7,10,13]$. When the input functions $h(y)$ are smooth, and the kernels $k(x, y)$ satisfy certain conditions, these equations have solutions which are smooth everywhere in $(-1,1)$ except at the endpoints \pm 1 .

Among the numerical methods proposed to solve such equations we recall the Nyström methods, which are based on quadrature formulas. In particular, if we decide to discretize the integral

$$
\int_{-1}^{1} k(x, y) u(x) d x
$$

Received by the editor January 30, 1992 and, in revised form, November 6, 1992.

1991 Mathematics Subject Classification. Primary 65D32, 41A25; Secondary 65R20.

This work was supported by the Ministero dell'Università e della Ricerca Scientifica e Tecnologica of Italy. 
by a product rule of interpolatory type, which integrates exactly the singular terms that may be present in $k(x, y)$, then in order to derive accurate convergence estimates for the quadrature rule it is important to take into account the precise behavior of $u(x)$ in $[-1,1]$. We recall that once the stability of the Nyström method has been proved, let us say, in $C[-1,1]$, then the (uniform) norm of the error associated with the Nyström interpolant is bounded, up to a constant factor, by the norm of the quadrature error.

For a few examples of kernels such as $|x-y|^{\nu},-1<\nu<0$, and $\log |x-y|$, explicit expansions of $u(x)$ in $[-1,1]$ as a finite linear combination of singular terms plus a function which is smooth in $[-1,1]$ are known (see $[8,18])$. Since the singular terms of such expansions are of the form $(1 \pm x)^{k(1+\nu)}$ and $(1 \pm x)^{j} \log ^{l}(1 \pm x), l \leq j, k, j, l=1,2, \ldots$, in $[2,12,14]$ it has been sufficient to consider the behavior of the quadrature rules when they were applied to those terms.

There are however problems of type (1.1) where the kernel $k(x, y)$ is neither one of those above, nor a linear combination of them such as in [12]. Consider for instance a problem where $k(x, y)=|x-y|^{\nu} \log |x-y|$. In these situations we have not an explicit expansion of $u(x)$ in $[-1,1]$. Indeed, we can only state the following (see [22]):

Theorem 1. Consider (1.1) with $k(x, y) \equiv k(|x-y|)^{1}$ and $k(1+t) \in C^{q-1}(-1,1]$ and $h \in C^{q}[-1,1]$ for $q \geq 1$. Further assume that

$$
\begin{array}{ll}
\left|k^{(i)}(1+t)\right| \leq \gamma_{i}(1+t)^{-\alpha-i}, & -1<t \leq 1, \quad i=0,1, \ldots, q-1, \\
\left|k^{(i)}(1+t)\right| \geq \delta_{i}(1+t)^{-\alpha_{0}-i}, & -1<t \leq t_{0}, \quad i=0,1, \ldots, q-1,
\end{array}
$$

where $\alpha, \alpha_{0}$ are real constants such that

$$
0<\alpha<1 \text { and }-1<\alpha_{0} \leq \alpha,
$$

and $\gamma_{i}, \delta_{i}$ are some positive real constants and $-1<t_{0}<1$. If the homogeneous equation corresponding to $(1.1)$ has in $C[-1,1]$ only the trivial solution, then equation (1.1) has a unique solution $u(x) \in C[-1,1] \cap C^{q}(-1,1)$, and

$$
\begin{array}{ccc}
\left|u^{(i)}(x)\right| \leq \eta_{i}\left[(1+x)^{-\alpha-i+1}+(1-x)^{-\alpha-i+1}\right], \quad-1<x<1, & i=0,1, \ldots, q, \\
u^{(i)}(x)=u(-1) k^{(i-1)}(1+x)-u(1) k^{(i-1)}(1-x)+v_{i}(x), & i=1, \ldots, q,
\end{array}
$$

where $\eta_{i}, i=0,1, \ldots, q$, are positive constants, $v_{i} \in C^{q-i}(-1,1)$, and

$$
\lim _{x \rightarrow-1^{+}} \frac{v_{i}(x)}{k^{(i-1)}(1+x)}=0, \quad \lim _{x \rightarrow 1^{-}} \frac{v_{i}(x)}{k^{(i-1)}(1-x)}=0 .
$$

Notice that this theorem states that for the class of kernels considered, the corresponding solutions $u(x)$ belong to $C[-1,1] \cap C^{q}(-1,1), q \geq 1$; furthermore,

$$
\lim _{x \rightarrow \mp 1^{ \pm}}(1 \pm x)^{i} u^{(i)}(x)=0, \quad i=1, \ldots, q .
$$

By continuity we can thus define the functions $\left(1-x^{2}\right)^{i} u^{(i)}(x)$ also at $x= \pm 1$, so that $\left(1-x^{2}\right)^{i} u^{(i)}(x) \in C[-1,1], i=0,1, \ldots, q$, and set $u \in \bar{C}_{0}^{q}[-1,1]$,

\footnotetext{
${ }^{1}$ In [22] the authors state that without any serious difficulties one can generalize this theorem to the case of kernels $k(x, y)=m(x, y) k(|x-y|)$ or $k(x, y)=m(x, y) k(x-y)$, with $m(x, y)$ smooth.
} 
with

$\bar{C}_{p}^{q}[-1,1]=\left\{g \in L^{1}(-1,1):\left(1-x^{2}\right)^{i-p} g^{(i)}(x) \in C[-1,1], i=0,1, \ldots, q\right\}$.

We also remark that in the particular case examined in [2] there were no advantages in writing the function $u(x)$ in the form $(1-x)^{\alpha}(1+x)^{\beta} v(x)$ and considering $w(x)=(1-x)^{\alpha}(1+x)^{\beta}$ as a weight function, unless $v(x)$ was itself of class $C^{q}[-1,1]$; but this is not the case for the equations we are considering.

Finally, we recall that in the case of certain classes of Mellin convolution equations of the form $(1.1)$ (see $[1,7,13])$ invertible in $L^{\infty}(-1,1)$, when ${ }^{2}$ $h \in C_{p}^{q}[-1,1]+\Pi_{d}$ for some $q \geq 1$ and $p \geq 0$, where

$C_{p}^{q}[-1,1]=\left\{g \in L^{1}(-1,1):(1-x)^{i-p} g^{(i)}(x) \in C[-1,1], i=0,1, \ldots, q\right\}$,

we have $u \in C_{p}^{q}[-1,1]+\Pi_{d}$.

In this paper we are concerned with the discretization of integrals of the form

$$
\int_{-1}^{1} k(x, y) f(x) d x
$$

by means of quadrature formulas of interpolatory type based on the zeros of Jacobi orthogonal polynomials. These rules integrate exactly the singular components of the kernels (see, for example, $[2,10,12])$. To derive accurate uniform convergence estimates for these quadratures, we need to take into account the precise behavior of $f(x)$ in the interval of integration. This means that, if we refer to the cases mentioned in this introduction, we need to consider functions $f(x) \in \bar{C}_{p}^{q}[-1,1]$ or $f(x) \in C_{p}^{q}[-1,1]$, with $p \geq 0$ and $q>p$.

The starting point of our analysis is the search of accurate polynomial approximations for functions $f(x)$ of the type above.

Throughout this paper the symbol " $c$ " present in the bounds we state or derive will stand for a positive constant taking different values on different occurrences.

The well-known Jackson estimate

$$
\left\|f-P_{m}\right\|_{\infty} \leq c \omega_{r}\left(f ; m^{-1}\right),
$$

where

$$
\omega_{r}(f ; t)=\sup _{0<h \leq t}\left\|\Delta_{h}^{r} f\right\|_{[-1,1-r h]}
$$

and

$$
\omega_{r}(f ; t) \leq c t \omega_{r-1}\left(f^{\prime} ; t\right),
$$

for the uniform polynomial approximation of a continuous function on $[-1,1]$, is unsatisfactory when our function $f$ has some kind of irregularities at the endpoints \pm 1 , but is smooth everywhere inside $(-1,1)$; the uniform bound (1.3) must take into account also the behavior of $f(x)$ at \pm 1 . In such a situation estimates like those given in [5, §7.2]:

$$
E_{m}(f)_{p}=\inf _{P_{m} \in \Pi_{m}}\left\|f-P_{m}\right\|_{p} \leq c \omega_{\phi}^{r}\left(f, m^{-1}\right)_{p}, \quad m>r
$$

where we assume $f \in L^{p}(-1,1), 1 \leq p \leq \infty, \phi(x)=\sqrt{1-x^{2}}$, and where

$$
\omega_{\phi}^{r}\left(f, m^{-1}\right)_{p}=\sup _{0<h \leq m^{-1}}\left\|\Delta_{h \phi}^{r} f\right\|_{p},
$$

${ }^{2} \Pi_{d}$ denotes the space of polynomials of degree $d$. 
appear more suitable. Incidentally, if in (1.4) we take $r=1, \phi \equiv 1$ and $p=\infty$, we obtain (1.3). We also recall (see $[5, \S 4.2])$ that when $f^{(r-1)}(x)$ is absolutely continuous in any $[a, b] \subset(-1,1)$ and $\left\|\phi^{r} f^{(r)}\right\|_{p}<\infty$, we have $\omega_{\phi}^{r}(f ; t)=O\left(t^{r}\right)$; hence

$$
E_{m}(f)_{p}=O\left(m^{-r}\right) .
$$

However, this too was not sufficient for the applications we have considered in [2] and [10]. In these papers we had to derive some new pointwise and weighted $L^{1}$-polynomial approximation error estimates for the functions $f(x)=(1-x)^{\sigma}$ and $f(x)=(1-x)^{\sigma} h(x),\left|(1-x)^{k} h^{(k)}(x)\right| \leq c, k=0,1, \ldots, q$, respectively.

Results recently obtained by V. Totik in [21], although they could be applied to the function $f(x)=(1-x)^{\sigma}$ above, when $\sigma>0$, will not be sufficient for our present purposes. Indeed, in that paper Totik, proving a conjecture stated in [11], has shown that when the given function $f(x) \in C^{p}[-1,1]$ is analytic in $D=\{z \mid \operatorname{dist}(z,[-1,1])>c \operatorname{dist}(z, 1)\}$ there exists a sequence of algebraic polynomials $\left\{P_{m}(x)\right\}$ such that at each point $x \in(-1,1)$ of analyticity

$$
\left|P_{m}(x)-f(x)\right| \leq c_{f, x} e^{-m \sqrt{1-x}},
$$

where $c_{f, x}$ is bounded in each closed subinterval $[-1, c] \subset[-1,1)$, and

$$
\left\|f-P_{m}\right\|_{\infty} \leq \frac{\omega_{D}\left(f^{(2 p)} ; m^{-2}\right)}{m^{2 p}}
$$

where

$$
\omega_{D}(g ; t)=\sup \{|g(u)-g(v)|: u, v \in D \text { and }|u-v| \leq t\} .
$$

In the next section we generalize our polynomial approximation presented in [2] and [10] to include functions of class $\bar{C}_{p}^{q}[-1,1]$ and $C_{p}^{q}[-1,1]$, respectively, with $p$ an integer $\geq-1$ and $q$ an integer $>\max \{0, p\}$.

In particular, we derive some pointwise and uniform, as well as certain weighted $L^{1}$, polynomial approximation error estimates for functions with the properties stated above. These estimates appear to be new and of interest. They are useful to obtain error bounds for our quadrature rules. As an example of how one can proceed, in $\S 3$ we will first consider the case $|k(x, y)| \leq c|x-y|^{\nu}$, $-1<\nu<0$; then we will examine the case

$k(x, y)=k^{*}\left(\frac{1-y}{1-x}\right) \frac{1}{1-x}$, with $\left|k^{*}\left(\frac{1-y}{1-x}\right)\right| \leq c,-1 \leq x<1,|y| \leq 1$, associated with the Mellin equations previously mentioned.

2. POINTWISE AND UNIFORM POLYNOMIAL APPROXIMATION ERROR ESTIMATES

Lemma 1 (see [4]). Let $f(x) \in C^{r}[-1,1], r \geq 0$. Then, for each integer $m \geq 4 r+4$ there exists ${ }^{3}$ an algebraic polynomial $Q_{m}(x)$ of degree $m$ such that

$$
\left|f^{(k)}(x)-Q_{m}^{(k)}(x)\right| \leq c\left(\frac{\sqrt{1-x^{2}}}{m}\right)^{r-k} \omega_{2}\left(f^{(r)} ; \frac{\sqrt{1-x^{2}}}{m}\right), \underset{-1 \leq x \leq 1,}{ }
$$

\footnotetext{
${ }^{3}$ And it is explicitly defined.

${ }^{4}$ We recall that $\omega_{2}(g ; \delta) \leq 2 \omega(g ; \delta)$.
} 
for $k=0,1, \ldots, r$, the constant $c$ being independent of $x, m$, and $f$.

Lemma 2. Let $f(x) \in C^{r}[-1,1], r \geq 0$. For each integer $m \geq 4 r+4$ there exists ${ }^{5}$ an algebraic polynomial $P_{m}(x)$ of degree $m$ such that

$$
\left|f^{(k)}(x)-P_{m}^{(k)}(x)\right| \leq c\left(\frac{\sqrt{1-x^{2}}}{m}\right)^{r-k} E_{m-r}\left(f^{(r)}\right), \quad-1 \leq x \leq 1,
$$

for $k=0,1, \ldots, r$, where $E_{m-r}\left(f^{(r)}\right)$ denotes the uniform best polynomial approximation error (of degree $m-r$ ) associated with the function $f^{(r)}(x)$, $-1 \leq x \leq 1$, and $c$ is independent of $x, m$, and $f$.

Proof. Let $\bar{q}_{m-r}(x)$ be the (uniform) best approximation polynomial, of degree $m-r$, to $f^{(r)}$. Furthermore, let $q_{m}(x)$ be a polynomial of degree $m$ such that $q_{m}^{(r)}(x)=\bar{q}_{m-r}(x)$; we have

$$
\left\|f^{(r)}-q_{m}^{(r)}\right\|_{\infty}=E_{m-r}\left(f^{(r)}\right) .
$$

Since $f-q_{m} \in C^{r}[-1,1]$, by Lemma 1 we know there exists a polynomial $Q_{m}(x)$, of degree $m \geq 4 r+4$, such that

$$
\begin{gathered}
\left|f^{(k)}(x)-q_{m}^{(k)}(x)-Q_{m}^{(k)}(x)\right| \leq c\left(\frac{\sqrt{1-x^{2}}}{m}\right)^{r-k} \omega_{2}\left(f^{(r)}-q_{m}^{(r)} ; \frac{\sqrt{1-x^{2}}}{m}\right) \\
\quad \leq c\left(\frac{\sqrt{1-x^{2}}}{m}\right)^{r-k}\left\|f^{(r)}-q_{m}^{(r)}\right\|_{\infty}=c\left(\frac{\sqrt{1-x^{2}}}{m}\right)^{r-k} E_{m-r}\left(f^{(r)}\right)
\end{gathered}
$$

for $-1 \leq x \leq 1, k=0,1, \ldots, r$, and where the constant $c$ is independent of $x, m, f$. To obtain (2.2), we simply set $P_{m}(x)=q_{m}(x)+Q_{m}(x)$.

In the following we will need to consider the two auxiliary functions

$$
\begin{array}{ll}
\boldsymbol{\Phi}(x)=(1-x)^{q-p} f(x), & f \in C_{p}^{q}[-1,1], \\
\overline{\boldsymbol{\Phi}}(x)=\left(1-x^{2}\right)^{q-p} f(x), & f \in \bar{C}_{p}^{q}[-1,1] .
\end{array}
$$

Notice that we have

$$
\Phi^{(k)}(1)=0 \text { for } k=0,1, \ldots, q-p-1
$$

and

$$
\overline{\boldsymbol{\Phi}}^{(k)}( \pm 1)=0 \text { for } k=0,1, \ldots, q-p-1 .
$$

Then we have the following results.

Theorem 2. Let $f \in C_{p}^{q}[-1,1], p \geq-1, q>\max \{0, p\}$. Then there exists $a$ sequence of algebraic polynomials $t_{m}(x)$ such that for all integers $m$ sufficiently large

$$
\begin{array}{r}
\left|f(x)-t_{m}(x)\right| \leq c \omega_{2}\left(\Phi^{(q)} ; \frac{\sqrt{1-x^{2}}}{m}\right)\left(\frac{\sqrt{1+x}}{m}\right)^{q}(\sqrt{1-x})^{2 p-q} \\
-1 \leq x \leq 1
\end{array}
$$

\footnotetext{
${ }^{5}$ And it is explicitly defined.
} 
if $q \leq 2 p(p>0)$, and

$$
\begin{array}{r}
\left|f(x)-t_{m}(x)\right| \leq c \omega_{2}\left(\Phi^{(q)} ; \frac{\sqrt{1-x^{2}}}{m}\right)\left(\frac{\sqrt{1+x}}{m}\right)^{q} \frac{1}{(\sqrt{1-x})^{q-2 p}} \\
-1 \leq x<1,
\end{array}
$$

when $q \geq 2 p+1$. Furthermore, in this latter case we have

$$
\left|f(x)-t_{m}(x)\right| \leq c \frac{\omega_{2}\left(\Phi^{(q)} ; \frac{\sqrt{1-x^{2}}}{m}\right)}{1-x}, \quad 1-c m^{-2} \leq x<1,
$$

if $p=-1$, and

$$
\begin{aligned}
\left|f(x)-t_{m}(x)\right| \leq c \omega_{2}\left(\Phi^{(q)} ; \frac{\sqrt{1-x^{2}}}{m}\right)\left(\frac{\sqrt{1-x^{2}}}{m}\right)^{p}, \\
1-c m^{-2} \leq x \leq 1,
\end{aligned}
$$

if $p \geq 0$.

Proof. From Lemma 1 we know that for each integer $m \geq 4 q+4$ there exists a polynomial $Q_{m}(x)$ of degree $m$ such that

$$
\begin{aligned}
\left|\Phi^{(k)}(x)-Q_{m}^{(k)}(x)\right| \leq c\left(\frac{\sqrt{1-x^{2}}}{m}\right)^{q-k} \omega_{2}\left(\Phi^{(q)} ; \frac{\sqrt{1-x^{2}}}{m}\right), \\
k=0,1, \ldots, q .
\end{aligned}
$$

Moreover, since $\Phi^{(k)}(1)=0$ for $k=0,1, \ldots, q-p-1$, we also have

$$
Q_{m}^{(k)}(1)=\Phi^{(k)}(1)=0 \quad k=0,1, \ldots, q-p-1 ;
$$

hence,

$$
Q_{m}(x)=(1-x)^{q-p} t_{m+p-q}(x) .
$$

Inserting this latter expression into (2.7) with $k=0$, and recalling that $\Phi(x)=$ $(1-x)^{q-p} f(x)$, we obtain the inequality

$$
\begin{array}{r}
\left|f(x)-t_{m+p-q}(x)\right| \leq c \omega_{2}\left(\Phi^{(q)} ; \frac{\sqrt{1-x^{2}}}{m}\right)(\sqrt{1-x})^{2 p-q}\left(\frac{\sqrt{1+x}}{m}\right)^{q}, \\
-1<x<1,
\end{array}
$$

from which we easily derive (2.3) and (2.4).

To obtain the bound in (2.5), we use the relation

$$
\left|f(x)-t_{m-1-q}(x)\right|=\frac{\left|\Phi(x)-Q_{m}(x)\right|}{(1-x)^{q+1}}=\frac{\left|\Phi^{(q)}\left(\xi_{x}\right)-Q_{m}^{(q)}\left(\xi_{x}\right)\right|}{q !(1-x)}, \quad x<\xi_{x}<1,
$$

and apply (2.7). In the case $p \geq 0$, we proceed similarly to obtain (2.6). In particular, we start from the expression

$$
\left|f(x)-t_{m+p-q}(x)\right|=\frac{\left|\Phi^{(q-p)}\left(\xi_{x}\right)-Q_{m}^{(q-p)}\left(\xi_{x}\right)\right|}{(q-p) !}, \quad x<\xi_{x}<1 .
$$


Corollary 1. When in Theorem 2 we assume $p \geq 0$ and $q \geq 2 p+1$ we have

$$
\begin{array}{r}
\left|f(x)-t_{m}(x)\right| \leq c \frac{\omega\left(\Phi^{(q)} ; m^{-2}\right)}{m^{q-1}} \frac{(\sqrt{1+x})^{q}}{\left(\sqrt{1-x}+m^{-1}\right)^{q-2 p-1},} \\
-1 \leq x \leq 1 ;
\end{array}
$$

hence

$$
\left\|f-t_{m}\right\|_{\infty} \leq c \frac{\omega\left(\Phi^{(q)} ; m^{-2}\right)}{m^{2 p}} .
$$

Proof. When $-1 \leq x \leq-1+m^{-2}$, then (2.8) follows directly from (2.4). For $1-m^{-2} \leq x \leq 1$ it is sufficient to recall (2.6) and write

$$
\begin{aligned}
\left|f(x)-t_{m}(x)\right| & \leq c \frac{\omega\left(\Phi^{(q)} ; m^{-2}\right)}{m^{2 p}} \\
& \leq c \frac{\omega\left(\Phi^{(q)} ; m^{-2}\right)}{m^{q-1}} \frac{(\sqrt{1+x})^{q}}{\left(\sqrt{1-x}+m^{-1}\right)^{q-2 p-1}} .
\end{aligned}
$$

When $|x| \leq 1-m^{-2}$, from (2.4) we first derive

$$
\left|f(x)-t_{m}(x)\right| \leq c \frac{\omega\left(\Phi^{(q)} ; \frac{\sqrt{1-x^{2}}}{m}\right)}{\frac{\sqrt{1-x^{2}}}{m}} \frac{(\sqrt{1+x})^{q+1}}{m^{q+1}(\sqrt{1-x})^{q-2 p-1}} ;
$$

then, since

$$
\frac{\omega\left(f ; \delta_{2}\right)}{\delta_{2}} \leq \frac{\omega\left(f ; \delta_{1}\right)}{\delta_{1}}
$$

whenever $\delta_{2} \geq \delta_{1}$, by taking $\delta_{2}=\sqrt{1-x^{2}} / m$ and $\delta_{1}=m^{-2}$, and noting that

$$
\sqrt{1-x}=\frac{1}{2}[\sqrt{1-x}+\sqrt{1-x}] \geq \frac{1}{2}\left[\sqrt{1-x}+m^{-1}\right]
$$

we obtain

$$
\left|f(x)-t_{m}(x)\right| \leq c \frac{\omega\left(\Phi^{(q)} ; m^{-2}\right)}{m^{q-1}} \frac{(\sqrt{1+x})^{q+1}}{\left(\sqrt{1-x}+m^{-1}\right)^{q-2 p-1}},
$$

hence $(2.8)$.

The bound (2.9) follows immediately from (2.8).

Theorem 2'. Let $f \in \bar{C}_{p}^{q}[-1,1], p \geq-1, q>\max \{0, p\}$. Then there exists $a$ sequence of algebraic polynomials $t_{m}(x)$ such that for all integers $m$ sufficiently large

$$
\left|f(x)-t_{m}(x)\right| \leq c \omega_{2}\left(\bar{\Phi}^{(q)} ; \frac{\sqrt{1-x^{2}}}{m}\right) \frac{\left(\sqrt{1-x^{2}}\right)^{2 p-q}}{m^{q}}
$$

$$
-1 \leq x \leq 1
$$

if $q \leq 2 p \quad(p>0)$, and

$$
\begin{aligned}
&\left|f(x)-t_{m}(x)\right| \leq c \omega_{2}\left(\bar{\Phi}^{(q)} ; \frac{\sqrt{1-x^{2}}}{m}\right) \frac{1}{m^{q}\left(\sqrt{1-x^{2}}\right)^{q-2 p}} \\
&-1<x<1,
\end{aligned}
$$


when $q \geq 2 p+1$. Furthermore, in this latter case we have $\left(2.5^{\prime}\right)$

$$
\left|f(x)-t_{m}(x)\right| \leq c \omega_{2}\left(\bar{\Phi}^{(q)} ; \frac{\sqrt{1-x^{2}}}{m}\right) \begin{cases}\frac{1}{1-x}, & 1-c m^{-2} \leq x<1, \\ \frac{1}{1+x}, & -1<x \leq-1+c m^{-2},\end{cases}
$$

if $p=-1$, and

$$
\begin{gathered}
\left|f(x)-t_{m}(x)\right| \leq c \omega_{2}\left(\bar{\Phi}^{(q)} ; \frac{\sqrt{1-x^{2}}}{m}\right)\left(\frac{\sqrt{1-x^{2}}}{m}\right)^{p}, \\
1-c m^{-2} \leq|x| \leq 1,
\end{gathered}
$$

if $p \geq 0$.

Proof. The proof is very similar to that of Theorem 2.

If in the proof of Theorems 2 and $2^{\prime}$ we use Lemma 2 instead of Lemma 1, we can state the following alternative results.

Theorem 3. Under the hypotheses of Theorem 2 , for all integers $m$ sufficiently large there exists a sequence of algebraic polynomials $v_{m}(x)$ such that

$$
\begin{array}{r}
\left|f(x)-v_{m}(x)\right| \leq c E_{m-q}\left(\Phi^{(q)}\right)\left(\frac{\sqrt{1+x}}{m}\right)^{q}(\sqrt{1-x})^{2 p-q}, \\
-1 \leq x \leq 1,
\end{array}
$$

if $q \leq 2 p \quad(p>0)$, and

$$
\begin{aligned}
\left|f(x)-v_{m}(x)\right| \leq c E_{m-q}\left(\Phi^{(q)}\right)\left(\frac{\sqrt{1+x}}{m}\right)^{q} \frac{1}{(\sqrt{1-x})^{q-2 p}}, & \\
-1 \leq x<1, & \text {. }
\end{aligned}
$$

when $q \geq 2 p+1$. Furthermore, in this latter case, when $p \geq 0$, we have

$$
\left|f(x)-v_{m}(x)\right| \leq c E_{m-q}\left(\Phi^{(q)}\right)\left(\frac{\sqrt{1-x^{2}}}{m}\right)^{p}, \quad 1-c m^{-2} \leq x \leq 1 .
$$

Similarly, when $f \in \bar{C}_{p}^{q}[-1,1]$, we can derive the analogue of Theorem $2^{\prime}$ with $\omega_{2}\left(\overline{\boldsymbol{\Phi}}^{(q)} ; \frac{\sqrt{1-x^{2}}}{m}\right)$ replaced by $E_{m-q}\left(\overline{\boldsymbol{\Phi}}^{(q)}\right)$.

Theorem $3^{\prime}$. Under the hypotheses of Theorem $2^{\prime}$, for all integers $m$ sufficiently large there exists a sequence of algebraic polynomials $v_{m}(x)$ such that

$$
\left|f(x)-v_{m}(x)\right| \leq c E_{m-q}\left(\bar{\Phi}^{(q)}\right) \frac{\left(\sqrt{1-x^{2}}\right)^{2 p-q}}{m^{q}}, \quad-1 \leq x \leq 1,
$$

if $q \leq 2 p \quad(p>0)$, and

$$
\left|f(x)-v_{m}(x)\right| \leq c E_{m-q}\left(\bar{\Phi}^{(q)}\right) \frac{1}{m^{q}\left(\sqrt{1-x^{2}}\right)^{q-2 p}}, \quad-1<x<1,
$$

when $q \geq 2 p+1$. Furthermore, in this latter case, when $p \geq 0$, we have

$$
\left|f(x)-v_{m}(x)\right| \leq c E_{m-q}\left(\bar{\Phi}^{(q)}\right)\left(\frac{\sqrt{1-x^{2}}}{m}\right)^{p}, \quad 1-c m^{-2} \leq|x| \leq 1 .
$$


Corollary 2. If in Theorems 2 and $2^{\prime}$ we assume $q>p \geq 0$, then we have

$$
\left\|f-v_{m}\right\|_{\infty} \leq c \frac{E_{m-q}\left(\Phi^{(q)}\right)}{m^{q^{*}}}, \quad\left\|f-v_{m}\right\|_{\infty} \leq c \frac{E_{m-q}\left(\bar{\Phi}^{(q)}\right)}{m^{q^{*}}},
$$

respectively, where $q^{*}=\min \{q, 2 p\}$.

Remark 1. Given a general function $f \in C^{p}[-1,1], p \geq 1$, for the best uniform approximation polynomial $v_{m}^{*}(x)$, of degree $m$, we have the following bound (see [20]):

$$
\left\|f-v_{m}^{*}\right\|_{\infty} \leq c \frac{E_{m-p}\left(f^{(p)}\right)}{m^{p}} .
$$

Corollary 2 states that when $f \in C_{p}^{q}[-1,1]$ or $f \in \bar{C}_{p}^{q}[-1,1]$, with $q>p \geq 0$, our polynomials $v_{m}(x)$ defined in Theorems 3 and $3^{\prime}$ satisfy the even better bound (2.13). Actually, if $q \geq 2 p$, we have $\left\|f-v_{m}\right\|_{\infty}=o\left(m^{-2 p}\right)$. This result allows us to derive corresponding uniform error estimates for sequences of Lagrange interpolation polynomials. In particular, given a sequence of sets of $m$ distinct nodes $\left\{x_{1}^{(m)}, x_{2}^{(m)}, \ldots, x_{m}^{(m)}\right\}, m=1,2, \ldots$, and denoting by $\left\|L_{m}\right\|$ the Lebesgue constant associated with each set, i.e.,

$$
\left\|L_{m}\right\|=\max _{\|f\|_{\infty}=1}\left\|L_{m}(f)\right\|_{\infty},
$$

where $L_{m}(f) \equiv L_{m}(f ; x)$ is the $(m-1)$ st-degree Lagrange polynomial, we have

$$
\left\|f-L_{m}(f)\right\|_{\infty} \leq\left(\left\|L_{m}\right\|+1\right)\left\|f-v_{m}\right\|_{\infty} .
$$

If we consider sets of nodes with optimal-order Lebesgue constants $\left(\left\|L_{m}\right\|=\right.$ $O(\log m))$, we obtain

$$
\left\|f-L_{m}(f)\right\|_{\infty} \leq c \log m\left\|f-v_{m}\right\|_{\infty} .
$$

We recall that examples of such sets of nodes are the zeros of classical Jacobi polynomials $P_{m}^{(\alpha, \beta)}(x)$, with $\alpha, \beta \leq-\frac{1}{2}$, the Clenshaw abscissas $\left\{\cos \frac{k \pi}{m-1}\right.$, $k=0,1, \ldots, m-1\}$, and the extensions of the zeros of Jacobi polynomials recently introduced in $[3,9]$.

\section{3. $L^{1}$-ERROR BOUNDS AND QUADRATURE ERRORS}

Using some of the estimates presented in the previous section, in the next two theorems we derive bounds for some corresponding weighted $L^{1}$-polynomial approximations. These bounds will be needed to obtain convergence error estimates for our product formulas.

First we consider the case $|k(x, y)| \leq c|x-y|^{\nu},-1<\nu<0$.

Theorem 4. Let $f \in \bar{C}_{p}^{q}[-1,1], p \geq 0, q>2 p+2+2 \nu,-1<\nu<0$. Then the polynomial $v_{m}(x)$ defined in Theorem $3^{\prime}$ satisfies the inequality

$$
\begin{aligned}
\int_{-1}^{1}\left|f(x)-v_{m}(x)\right||x-y|^{\nu} d x \\
\quad \leq \begin{cases}\frac{c}{m^{2 p+2+2 \nu}} E_{m-q}\left(\bar{\Phi}^{(q)}\right) & \text { if }|y| \leq 1, \\
\frac{c}{m^{2 p+2}} E_{m-q}\left(\bar{\Phi}^{(q)}\right) & \text { if }-1<c_{1} \leq y \leq c_{2}<1,\end{cases}
\end{aligned}
$$

where $\bar{\Phi}(x)=\left(1-x^{2}\right)^{q-p} f(x)$. 
Proof. For simplicity we consider only the case $-1 \leq y \leq 1$. First we decompose the integral in (3.1) as follows:

$$
\int_{-1}^{1}|x-y|^{\nu}\left|f(x)-v_{m}(x)\right| d x=\int_{-1}^{-1+m^{-2}}+\int_{-1+m^{-2}}^{1-m^{-2}}+\int_{1-m^{-2}}^{1}=: I_{1}+I_{2}+I_{3} .
$$

To bound $I_{1}$, we use $\left(2.12^{\prime}\right)$ :

$$
I_{1} \leq c \frac{E_{m-q}\left(\bar{\Phi}^{(q)}\right)}{m^{2 p}} \int_{-1}^{-1+m^{-2}}|x-y|^{\nu} d x \leq \frac{c}{m^{2 p+2+2 \nu}} E_{m-q}\left(\overline{\boldsymbol{\Phi}}^{(q)}\right) .
$$

Inserting $\left(2.11^{\prime}\right)$ into $I_{2}$ and using an argument similar to the one near the end of the proof of Corollary 1 , we get

$$
I_{2} \leq c \frac{E_{m-q}\left(\bar{\Phi}^{(q)}\right)}{m^{q}} \int_{-1}^{1}\left(\sqrt{1-x^{2}}+m^{-1}\right)^{2 p-q}|x-y|^{\nu} d x ;
$$

hence, recalling $[2 \text {, Lemma } 1]^{6}$, we obtain (3.1). The treatment of $I_{3}$ is analogous to that of $I_{1}$.

Theorems 5 and 6 below generalize corresponding results presented in $[2,14]$ in two ways. First, they apply to the more general function $f(x) \in \bar{C}_{p}^{q}[-1,1]$, and second, by adding to the Jacobi abscissas appropriate extra nodes, they allow the choice of any $\alpha, \beta>-1$ (in $[2,14]$ we have the condition $-\frac{1}{2} \leq \alpha, \beta \leq \frac{3}{2}$ ).

We denote by

$$
L_{m, 0,0}(f ; x)=\sum_{k=1}^{m} l_{m, k}(x) f\left(x_{k}\right)
$$

the $(m-1)$ st-degree Lagrange polynomial which interpolates the function $f(x)$ at the zeros $\left\{x_{k} \equiv x_{m, k}^{(\alpha, \beta)}\right\},-1<x_{1}<x_{2}<\cdots<x_{m}<1$, of the (orthonormal) Jacobi polynomial $P_{m}^{(\alpha, \beta)}(x)$. Furthermore, having chosen, for example (see [9]), $s$ equidistant nodes $\left\{y_{i}\right\}$ in $\left[-1, x_{1}\right)$ and $r$ equidistant nodes $\left\{z_{j}\right\}$ in $\left(x_{m}, 1\right]$, we denote by

$$
\begin{aligned}
L_{m, r, s}(f ; x)= & A_{s}(x) B_{r}(x) \sum_{k=1}^{m} l_{m, k}(x) \frac{f\left(x_{k}\right)}{A_{s}\left(x_{k}\right) B_{r}\left(x_{k}\right)} \\
& +B_{r}(x) P_{m}^{(\alpha, \beta)}(x) L_{s}\left(\frac{f}{B_{r} P_{m}^{(\alpha, \beta)}} ; x\right) \\
& +A_{s}(x) P_{m}^{(\alpha, \beta)}(x) L_{r}\left(\frac{f}{A_{s} P_{m}^{(\alpha, \beta)}} ; x\right),
\end{aligned}
$$

where

$$
A_{s}(x)=\prod_{i=1}^{s}\left(x-y_{i}\right), \quad B_{r}(x)=\prod_{j=1}^{r}\left(x-z_{j}\right),
$$

${ }^{6}$ We have $p-\frac{q}{2}+1+\nu<0$. 
the Lagrange polynomial of degree $m+r+s-1$ which interpolates the function $f(x)$ at the new set of nodes

$$
-1 \leq y_{1}<\cdots<y_{s}<x_{1}<x_{2}<\cdots<x_{m}<z_{1}<\cdots<z_{r} \leq 1 .
$$

In (3.3), $L_{s}(g ; x)$ and $L_{r}(g ; x)$ are the Lagrange polynomials which interpolate the function $g$ at the abscissas $\left\{y_{i}\right\}$ and $\left\{z_{j}\right\}$, respectively.

Theorem 5. Let $f \in \bar{C}_{p}^{q}[-1,1]$, with $p \geq 0$ and $q>2 p+2+2 \nu$. If in (3.3) we assume $r \geq 0, s \geq 0$ such that

$$
-\frac{3}{2}-2 \nu<2 r-\alpha, \quad 2 s-\beta \leq \frac{1}{2}-2 \nu,
$$

then we have

$$
\begin{aligned}
& \int_{-1}^{1}\left|f(x)-L_{m, r, s}(f ; x)\right||x-y|^{\nu} d x \\
& \quad \leq c \frac{E_{m-q-1}\left(\bar{\Phi}^{(q)}\right) \log m}{m^{2 p+2}} \begin{cases}\frac{1}{m^{2 \nu}} & \text { if }|y| \leq 1, \\
1 & \text { if }-1<c_{1} \leq y \leq c_{2}<1 .\end{cases}
\end{aligned}
$$

Proof. For simplicity we consider only the case $-1 \leq y \leq 1$. Furthermore, since $L_{m, 0,0}(f ; x)$ can be considered as a special case of $L_{m, r, s}(f ; x)$, we will refer only to this latter.

Since the interpolation operator $L_{m, r, s}$ reproduces polynomials of degree $m-1$, we can write

$$
\begin{aligned}
& \int_{-1}^{1}\left|f(x)-L_{m, r, s}(f ; x)\right||x-y|^{\nu} d x \\
& \quad \leq \int_{-1}^{1}\left|f(x)-v_{m}(x)\right||x-y|^{\nu} d x+\int_{-1}^{1}\left|L_{m, r, s}\left(f-v_{m} ; x\right)\right||x-y|^{\nu} d x,
\end{aligned}
$$

where $v_{m}(x)$ is the polynomial of degree $m-1$ defined in Theorem $3^{\prime}$. A bound for the first integral on the right-hand side is given in Theorem 4; the last integral needs some more work.

For any $x \in[-1,1]$, let $x_{c}=x_{c}(x)$ be the Jacobi node closest to $x$. Then write

$$
L_{m, r, s}\left(f-v_{m} ; x\right)=P_{1}+P_{2}+P_{3}+P_{4}
$$

with

$$
\begin{aligned}
& P_{1}=A_{s}(x) B_{r}(x) l_{m, c}(x) \frac{f\left(x_{c}\right)-v_{m}\left(x_{c}\right)}{A_{s}\left(x_{c}\right) B_{r}\left(x_{c}\right)}, \\
& P_{2}=A_{s}(x) B_{r}(x) \sum_{k=1, k \neq c}^{m} l_{m, k}(x) \frac{f\left(x_{k}\right)-v_{m}\left(x_{k}\right)}{A_{s}\left(x_{k}\right) B_{r}\left(x_{k}\right)}, \\
& P_{3}=B_{r}(x) P_{m}^{(\alpha, \beta)}(x) L_{s}\left(\frac{f-v_{m}}{B_{r} P_{m}^{(\alpha, \beta)}} ; x\right), \\
& P_{4}=A_{s}(x) P_{m}^{(\alpha, \beta)}(x) L_{r}\left(\frac{f-v_{m}}{A_{s} P_{m}^{(\alpha, \beta)}} ; x\right) .
\end{aligned}
$$


Before proceeding further, we recall the following bounds, where here and below $C$ denotes a suitable positive constant taking different values on different occurrences:

$$
\begin{gathered}
C \leq \frac{A_{s}\left(x_{k}\right)}{\left(1+x_{k}\right)^{s}} \leq 1, \quad C \leq \frac{B_{r}\left(x_{k}\right)}{\left(1-x_{k}\right)^{r}} \leq 1, \\
\left|l_{m, c}(x)\right| \leq C \quad(\text { see }[15, \text { proof of Theorem 33, p. 171]), } \\
\left|l_{m, k}(x)\right| \leq C \frac{(1-x)^{\alpha / 2+3 / 4}(1+x)^{\beta / 2+3 / 4}}{m\left|x-x_{k}\right|}\left|P_{m}^{(\alpha, \beta)}(x)\right|, \quad k \neq c \\
\quad(\text { see [2, p. 225 ) } \\
\left|A_{s}(x)\right| \leq C\left(\sqrt{1+x}+m^{-1}\right)^{2 s}, \quad B_{r}(x) \mid \leq C\left(\sqrt{1-x}+m^{-1}\right)^{2 r} \\
\left|P_{m}^{(\alpha, \beta)}(x)\right| \leq C\left(\sqrt{1-x}+m^{-1}\right)^{-\alpha-1 / 2}\left(\sqrt{1+x}+m^{-1}\right)^{-\beta-1 / 2}
\end{gathered}
$$

$$
\begin{aligned}
& \prod_{i=1, i \neq j}^{s}\left|\frac{x-y_{i}}{y_{j}-y_{i}}\right| \leq C m^{2 s-2}\left(\sqrt{1+x}+m^{-1}\right)^{2 s-2}, \\
& \prod_{j=1, j \neq i}^{r}\left|\frac{x-z_{j}}{z_{i}-z_{j}}\right| \leq C m^{2 r-2}\left(\sqrt{1-x}+m^{-1}\right)^{2 r-2} .
\end{aligned}
$$

Now let us consider $P_{1}$ in (3.5):

$$
P_{1}=A_{s}(x) B_{r}(x) l_{m, c}(x) \frac{f\left(x_{c}\right)-v_{m}\left(x_{c}\right)}{A_{s}\left(x_{c}\right) B_{r}\left(x_{c}\right)} ;
$$

after noticing that $\left|\frac{A_{s}(x) B_{r}(x)}{A_{s}\left(x_{c} B_{r}\left(x_{c}\right)\right.}\right| \leq C$, recalling (3.7) and (2.11'), we obtain

$$
\begin{aligned}
\left|P_{1}\right| & \leq C \frac{E_{m-q-1}\left(\bar{\Phi}^{(q)}\right)}{m^{q}} \frac{1}{\left(\sqrt{1-x_{c}^{2}}\right)^{q-2 p}} \\
& \leq C \frac{E_{m-q-1}\left(\bar{\Phi}^{(q)}\right)}{m^{q}} \frac{1}{\left(\sqrt{1-x^{2}}+m^{-1}\right)^{q-2 p}}, \quad-1 \leq x<1 ;
\end{aligned}
$$

hence

$$
\int_{-1}^{1}\left|P_{1}\right||x-y|^{\nu} d x \leq C \frac{\log m}{m^{2 p+2+2 \nu}} E_{m-q-1}\left(\bar{\Phi}^{(q)}\right) .
$$

In the case of $P_{2}$, by (3.6), (3.8), (3.9), (3.10), and (2.11') we have

$$
\begin{aligned}
\left|P_{2}\right| \leq & C\left(\sqrt{1-x}+m^{-1}\right)^{-\alpha-1 / 2+2 r}\left(\sqrt{1+x}+m^{-1}\right)^{-\beta-1 / 2+2 s} \\
& \cdot \frac{E_{m-q-1}\left(\bar{\Phi}^{(q)}\right)}{m^{q}} \sum_{k=1, k \neq c}^{m} \frac{\left(1+x_{k}\right)^{\beta / 2+3 / 4-s-q / 2+p}\left(1-x_{k}\right)^{\alpha / 2+3 / 4-r-q / 2+p}}{m\left|x-x_{k}\right|} .
\end{aligned}
$$

The latter sum can be bounded by

$$
C\left[\sum_{k=1, k \neq c}^{m} \frac{\left(1+x_{k}\right)^{\beta / 2+3 / 4-s-q / 2+p}}{m\left|x-x_{k}\right|}+\sum_{k=1, k \neq c}^{m} \frac{\left(1-x_{k}\right)^{\alpha / 2+3 / 4-r-q / 2+p}}{m\left|x-x_{k}\right|}\right] .
$$


Break the interval of integration in two parts: one from -1 to 0 , the other from 0 to 1 , and consider the corresponding two integrals ${ }^{7}$. By applying Lemmas 5 and 1 in [2], and examining all possible combinations of the expressions involved, we obtain

$$
\int_{-1}^{1}\left|P_{2}\right||x-y|^{\nu} d x \leq C \frac{\log m}{m^{2 p+2+2 \nu}} E_{m-q-1}\left(\bar{\Phi}^{(q)}\right) .
$$

To bound $P_{3}$, we first note that by (3.11) we have

$$
\left|L_{s}\left(\frac{f-v_{m}}{B_{r} P_{m}^{(\alpha, \beta)}} ; x\right)\right| \leq C m^{2 s-2}\left(\sqrt{1+x}+m^{-1}\right)^{2 s-2} \sum_{i=1}^{s} \frac{\left|f\left(y_{i}\right)-v_{m}\left(y_{i}\right)\right|}{\left|B_{r}\left(y_{i}\right) P_{m}^{(\alpha, \beta)}\left(y_{i}\right)\right|} ;
$$

then, recalling (3.9) and $\left(2.12^{\prime}\right)$, we obtain

$$
\left|P_{3}\right| \leq C \frac{E_{m-q-1}\left(\bar{\Phi}^{(q)}\right)}{m^{2 p}} m^{2 s-5 / 2-\beta}\left(\sqrt{1-x}+m^{-1}\right)^{2 r-\alpha-1 / 2}\left(\sqrt{1+x}+m^{-1}\right)^{2 s-\beta-5 / 2},
$$

since it is well known (see [16]) that $P_{m}^{(\alpha, \beta)}(x) \sim m^{\beta+1 / 2}$ when $-1 \leq x \leq$ $-1+\mathrm{Cm}^{-2}$. Finally, by applying Lemma 1 in [2], we derive

$$
\int_{-1}^{1}\left|P_{3}\right||x-y|^{\nu} d x \leq C \frac{\log m}{m^{2 p+2+2 \nu}} E_{m-q-1}\left(\bar{\Phi}^{(q)}\right) \text {. }
$$

The treatment of $P_{4}$ is very similar and leads to the same bound.

A second application of the results obtained in $\S 2$ is to the discretization of integrals of form (1.2) appearing in certain classes of Mellin convolution equations with $k(x, y)=k^{*}\left(\frac{1-y}{1-x}\right) \frac{1}{1-x}$, where $k^{*}(\cdot)$ is bounded. For examples of integral equations with kernels of this type, see for instance $[1,7,13]$.

In this case we consider integration rules based on $L_{m, r, s}(f ; x)$ with $r \geq 1$ and $z_{r}=1$. The proof of the following theorem is very similar to that of Theorem 5, with $\nu=0$ and the results of Theorem $3^{\prime}$ replaced by those of Theorem 3. We need only assume $p \geq 1$ and recall that now $B_{r}(x)$ has the factor $(1-x)$, which cancels the corresponding one in the denominator of $k(x, y)$, and that $f\left(z_{r}\right)-v_{m}\left(z_{r}\right)=0$ (see (2.12)). Indeed, $P_{1}, P_{2}, P_{3}$, and $P_{4}$ all have the factor $(1-x)$.

Theorem 6. Let $f \in C_{p}^{q}[-1,1]$, with $p \geq 1$ and $q \geq 2 p+1$. If in (3.3) we choose $r \geq 1, s \geq 0$ such that

and $z_{r}=1$, then we have

$$
\frac{3}{2}+q \geq 2 s-\beta \geq-\frac{3}{2} \text { and } 2 r-\alpha \leq \frac{5}{2},
$$

$$
\int_{-1}^{1}|k(x, y)|\left|f(x)-L_{m, r, s}(f ; x)\right| d x \leq C \frac{\log m}{m^{2 p}} E_{m-q-1}\left(\Phi^{(q)}\right) .
$$

Remark 2. In Theorem 6 we have made the hypothesis $p \geq 1$. However, similar results can be obtained under the weaker condition $p \geq 0$ if we are willing to use Theorem 2 and assume $\omega_{2}\left(\Phi^{(q)} ; \delta\right) \leq M \delta^{\lambda},-p<\bar{\lambda} \leq 2$. In particular, we would obtain a bound of the form $O\left(\log m / m^{2 p+2 \lambda}\right)$.

Some numerical evidence for the convergence results reported in Theorems 5 and 6 can be found in [12] and [10], respectively.

\footnotetext{
${ }^{7}$ Since $P_{m}^{(\alpha, \beta)}(-x)=(-1)^{m} P_{m}^{(\beta, \alpha)}(x)$, it is sufficient to consider only one of the two integrals; the other will be similar, with $\alpha$ and $\beta$ interchanged.
} 


\section{BIBLIOGRAPHY}

1. G. A. Chandler and I. G. Graham, Product integration-collocation methods for noncompact integral operator equations, Math. Comp. 50 (1988), 125-138.

2. G. Criscuolo, G. Mastroianni, and G. Monegato, Convergence properties of a class of product formulas for weakly singular integral equations, Math. Comp. 55 (1990), 213-230.

3. G. Criscuolo, G. Mastroianni, and P. Vértesi, Pointwise simultaneous convergence of extended Lagrange interpolation with additional knots, Math. Comp. 59 (1992), 515-531.

4. R. Dahlhaus, Pointwise approximation by algebraic polynomials, J. Approx. Theory 57 (1989), 274-277.

5. Z. Ditzian and V. Totik, Moduli of smoothness, Springer-Verlag, New York, 1989.

6. R. Duduchava, Integral equations with fixed singularities, Teubner Texte zur Math., vol. 24, Teubner Leipzig, 1979.

7. J. Elschner, Spline approximation methods for a class of non-compact integral equations, Report R-MATH-09/88, Akad. Wiss. DDR, Inst. Math., Berlin, 1988.

8. I. G. Graham, Singularity expansions for the solutions of second kind Fredholm integral equations with weakly singular convolution kernels, J. Integral Equations 4 (1982), 1-30.

9. G. Mastroianni, Uniform convergence of the derivatives of Lagrange interpolation, J. Comput. Appl. Math. 43 (1992), 37-51.

10. G. Mastroianni and G. Monegato, Nyström interpolants based on the zeros of Legendre polynomials for a non compact integral operator equation, IMA J. Numer. Anal., to appear.

11. G. Mastroianni and J. Szabados, Polynomial approximation of analytic functions with singularities, to appear.

12. G. Monegato and V. Colombo, Product integration for the linear transport equation in slab geometry, Numer. Math. 52 (1988), 219-240.

13. G. Monegato and S. Prössdorf, On the numerical treatment of an integral equation arising from a cruciform crack problem, Math. Methods Appl. Sci. 12 (1990), 489-502.

14. G. Monegato, Product integration for one-dimensional integral equations of Fredholm type, Atti Sem. Mat. Fis. Univ. Modena 34 (1991), 73-86.

15. P. Nevai, Orthogonal polynomials, Mem. Amer. Math. Soc. No. 213 (1979).

16. _ Mean convergence of Lagrange interpolation. III, Trans. Amer. Math. Soc. 282 (1984), 669-698.

17. R. Piessens, Modified Clenshaw-Curtis integration and applications to numerical computation of integral transforms, Numerical Integration (P. Keast and G. Fairweather, eds.), NATO Adv. Sci. Inst. Ser. C. Math. Phys. Sci., vol. 203, Reidel, Dordrecht, 1987, pp. 35-51.

18. J. Pitkäranta, On the differential properties of solutions to Fredholm equations with weakly singular kernels, J. Inst. Math. Appl. 24 (1979), 109-119.

19. B. Sendov and V. A. Popov, The averaged moduli of smoothness, Wiley, New York, 1989.

20. A. F. Timan, Theory of approximation of functions of a real variable (Russian). [English translation: Pergamon Press, The MacMillan Co., 1963.]

21. V. Totik, Polynomial approximation with locally geometric rate, Approximation Theory, Colloq. Math. Soc. János Bolyai, vol. 58, North-Holland, Amsterdam and New York, 1990, pp. 663-671.

22. G. Vainikko and A. Pedas, The properties of solutions of weakly singular integral equations, J. Austral. Math. Soc. Ser. B 22 (1981), 419-430.

Dipartimento di Matematica, Università della Basilicata, Potenza, Italia

E-mail address: mastroianniQperx85.cineca.it

Dipartimento di Matematica, Politecnico di Torino, Torino, Italia

E-mail address: monegato@itopoli.bitnet 